\title{
Measurements of the Lower Uterine Segment at Term in Women with Previous Cesarean Delivery
}

\author{
Tadatsugu Kinjo, Hitoshi Masamoto, Keiko Mekaru, Yusuke Taira, Yukiko Chinen, \\ Hayase Nitta, Yoichi Aoki \\ Department of Obstetrics and Gynecology, Graduate School of Medicine, University of the Ryukyus, Nishihara, \\ Okinawa, Japan \\ Email: yoichi@med.u-ryukyu.ac.jp
}

Received 8 December 2015; accepted 8 January 2016; published 11 January 2016

Copyright (C) 2016 by authors and Scientific Research Publishing Inc.

This work is licensed under the Creative Commons Attribution International License (CC BY). http://creativecommons.org/licenses/by/4.0/

(c) (i) Open Access

\begin{abstract}
Purpose: To evaluate the accuracy of sonographic measurements of the lower uterine segment (LUS) thickness at term in predicting uterine scar defects in women with previous Cesarean delivery (CD). Methods: Eighty-nine pregnant women who underwent $C D$ between 37 and 41 weeks of gestation from 2013 to 2015 were enrolled in this study and divided into two groups. Group A consisted of women with previous $C D$, and Group B consisted of women with previous vaginal deliveries. We performed an ultrasound evaluation of the myometrial and full thickness of LUS (mLUS and fLUS) transvaginally before a CD and evaluated the appearance of LUS during surgery, which was defined as follows: grade I, well-developed; grade II, thin without visible content; grade III, translucent with visible content; and grade IV, either dehiscence or rupture. Results: The median mLUS and fLUS were 1.50 and $4.07 \mathrm{~mm}$ in the group $\mathrm{A}$, and 2.75 and $5.37 \mathrm{~mm}$ in the group B. We observed significant differences in the median mLUS and fLUS between grades I/II $(2.07$ and $4.37 \mathrm{~mm})$ and grades III/IV $(0.67$ and $2.52 \mathrm{~mm})$. Both $\mathrm{mLUS}$ and fLUS were predictive factors for grades III/IV and cutoff values were $0.97 \mathrm{~mm}$ of mLUS and $3.13 \mathrm{~mm}$ of fLUS, having a sensitivity of $87.5 \%$ and $75.0 \%$, and a specificity of $87.7 \%$ and $91.4 \%$ in mLUS and fLUS measurement, respectively. Conclusion: Sonographic measurements of LUS at term may be a feasible and reliable method to predict uterine rupture or uterine dehiscence in women with prior CD.
\end{abstract}

\section{Keywords}

Lower Uterine Segment, Sonographic Measurement, Previous Cesarean, Uterine Rupture, Uterine Dehiscence 


\section{Introduction}

The rates of Cesarean delivery (CD) have increased in recent decades and continue to rise today. In Japan, it increased from $8 \%$ to $23 \%$ for hospital CDs and form 6\% to 13\% for clinic CDs from 1984 to 2008 [1]. On the contrary, a sharp and persistent decrease in vaginal birth after cesarean has been reported [2]. CDs are associated with severe obstetric complications in the following pregnancies, such as uterine rupture and placenta previa/ increta [3] [4], which can lead to catastrophic consequences for both mother and child. Furthermore, the increase in CDs and resultant escalation of medical costs will have a serious impact on the economy. Currently, the most frequent indication for CD is having a history for previous CD, which may weaken the lower uterine segment (LUS), leading to a uterine scar defect during pregnancies, especially during labor.

The National Institute of Health examined the evidence on maternal and neonatal outcomes in trials of labor after cesarean (TOLAC) and reached an evidence-based consensus statement that TOLAC remains a reasonable option for many women with a prior CD [5]. To decrease the rate of CD, the American College of Obstetricians and Gynecologists recommended that most pregnant women with a single previous low transverse CD be counseled and offered a TOLAC [6]. The risk of uterine rupture in laboring women with a previous CD varies between $0.2 \%$ and $1.5 \%$ after induction of labor compared with $0.5 \%$ in women with spontaneous labor after a previous CD [7] [8]. It is important in the counseling of TOLAC to inform the woman of her chance of success and discuss the maternal and neonatal risks and benefits.

Several studies have proposed that thinning in the LUS measured by ultrasonography is a predictor of uterine rupture [9]-[13]. However, an ideal LUS thickness cutoff value that can be used in clinical practice in women with a scarred uterus could not be defined by these studies. Accurate prediction of uterine rupture would therefore be extremely valuable because it would allow women at low risk to proceed with a TOLAC, whereas women at a high risk of uterine rupture could undergo a planned CD.

We therefore aimed to evaluate the accuracy of ultrasonographic measurements of the LUS thickness at term in predicting uterine scar defects in women with a prior CD compared with women without a prior CD, and to determine whether a trial of labor could be offered safely or should not be offered.

\section{Materials and Methods}

\subsection{Subjects}

We conducted this study between October 2013 and August 2015 in the Department of Obstetrics and Gynecology, University of the Ryukyus Hospital. Pregnant women between 37 and 41 gestational weeks with or without a previous CD who attempted a planned CD were recruited into the study. Exclusion criteria were: placental abnormalities (abruption, accreta, previa), uterine leiomyomas, fetal anomalies, abnormal fluid volume (oligohydramnios or polyhydramnios), and uterine contractions. All patients provided written informed consent before enrollment. This study was conducted according to the principles stated in the 1964 Declaration of Helsinki and all subsequent revisions, and was approved by the Institutional Review Board of our university on September 18, 2013 (\#571). Eighty-nine pregnant women who underwent a planned CD between 37 and 41 weeks of gestation were enrolled and divided into two groups. Group A $(n=69)$ consisted of women with a previous CD who did not want to attempt a vaginal delivery and Group B $(n=20)$ consisted of women with previous vaginal deliveries and no uterine scar.

\subsection{Assessment of Ultrasonography}

We performed an ultrasonography of the myometrial thickness and full LUS thickness (mLUS and fLUS, respectively) transvaginally in the operating room before the women underwent a CD. The measurements were performed on Voluson-i machine (GE Healthcare, Milwaukee, WI, USA) using 5 - 9 MHz transvaginal probes by the same skilled sonographer (T.K.), according to the method described by Ginsberg et al., and Cheung et al. [14] [15]. The LUS thickness was evaluated using a transvaginal approach with an empty urinary bladder to ensure adequate visualization of the LUS. Sonographically, the normal LUS is a 2-layer structure that consists of an echogenic layer (including the bladder wall, fLUS) and a layer that is usually less echogenic (considered to represent the myometrium, mLUS) [15]. Once the area of myometrium was identified, the image was magnified up to two-thirds of the screen and captured as a still image, and calipers were used to measure the LUS thickness (Figure 1). The LUS was examined longitudinally and transversely, and the thinnest zone was identified and 


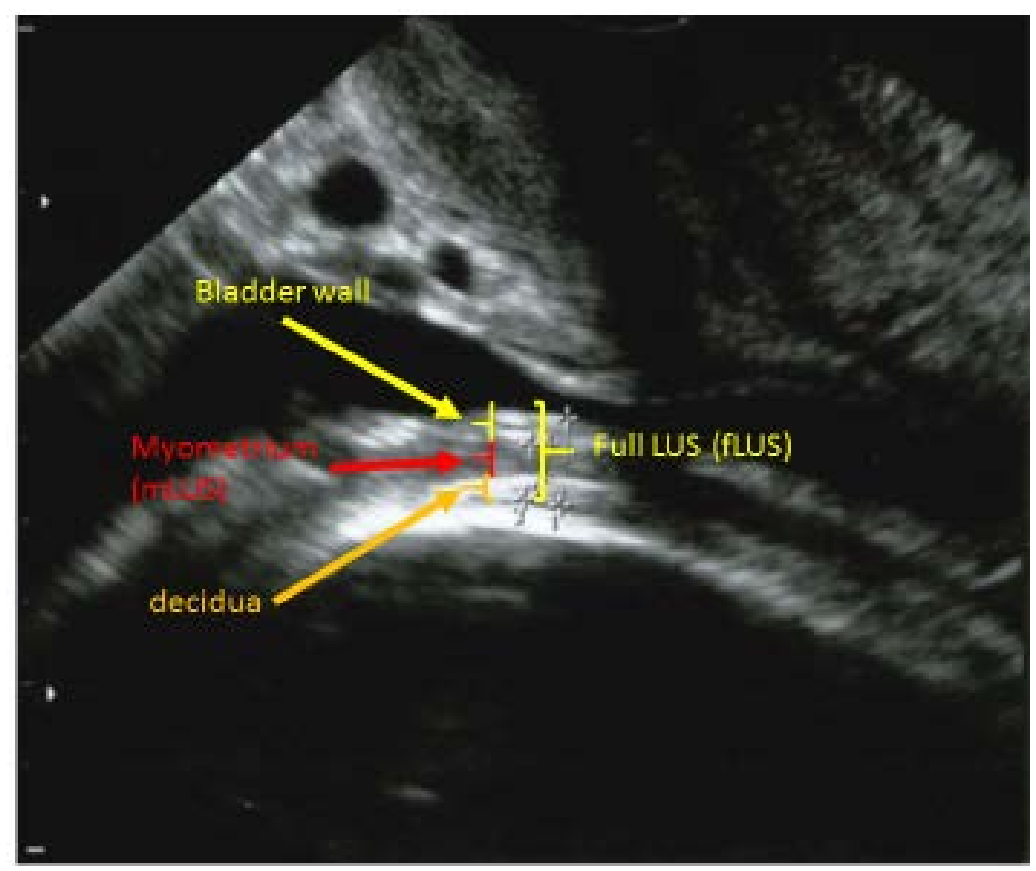

Figure 1. Sonograms show the measurement of the lower uterine segment.

quantified. At least 3 measurements were made, and the lowest value was retained as the dependent variable. During the CD, the surgeon objectively evaluated the LUS integrity and thickness according to the grading of the LUS as described by Qureshi et al. [16], which was defined as follows: grade I, well-developed; grade II, thin without visible content; grade III, translucent with visible content; and grade IV, either dehiscence or rupture. Ultrasonographic LUS thickness measurements were compared with the LUS grades.

\subsection{Statistical Analysis}

Statistical analyses were performed using JMP version 10.0.2 (SAS Institute Inc., Cary, NC, USA), using parametric and nonparametric tests when appropriate. Fisher's exact test, $\chi^{2}$ test, and Wilcoxon test were used. Univariate logistic regression analysis was used to identify the risk factors for grade III/IV LUS. Receiver operating characteristic analysis was applied for the selection of cutoff values of the LUS thickness. p values $<0.05$ were considered statistically significant.

\section{Results}

Baseline characteristics of both groups are shown in Table 1. No significant differences were observed between the groups except parity. The median number of previous CDs was 1 (range, $1-4$ ), and the median time from the last CD was 35 months (range, 14 - 156) in group A. The median mLUS thickness was $1.50 \mathrm{~mm}$ (range, 0.40 - 3.83) in group A and $2.75 \mathrm{~mm}$ (range, $0.77-10.7$ ) in group $B$, and median fLUS thickness was $4.07 \mathrm{~mm}$ (range, 1.53 - 7.03) in group A and $5.37 \mathrm{~mm}$ (range, 3.30 - 17.4) in group B: the differences between the groups were statistically significant $(\mathrm{p}<0.0001$ and $\mathrm{p}=0.0023$, respectively) (Table 2$)$.

Grades II-IV of LUS were observed only in group A, in which 35 patients were classified as grade I, 26 patients as grade II, 6 patients as grade III, and 2 patient as grade IV. Only grade I was observed in 20 patients of group B. We observed statistically significant differences in sonographic median mLUS thickness between grades I/II (2.07 mm, range, $0.40-10.7 \mathrm{~mm})$ and grades III/IV $(0.67 \mathrm{~mm}$, range, $0.40-1.47 \mathrm{~mm})(\mathrm{p}<0.0001)$ and in median fLUS thickness between grades I/II $(4.37 \mathrm{~mm}$, range, $1.53-17.4 \mathrm{~mm})$, and grades III/IV (2.52 $\mathrm{mm}$, range, $1.60-4.30 \mathrm{~mm})(\mathrm{p}=0.0005)$ (Table 3$)$. We found that both mLUS (odds ratio, 0.031 ; $95 \%$ confidence interval, 0.0016 - 0.219; $\mathrm{p}<0.0001$ ) and fLUS (odds ratio, 0.198; 95\% confidence interval, 0.059 - 0.481; $\mathrm{p}<0.0001$ ) were predictive factors by univariate logistic regression analysis (Table 4). There were no significant correlations between the performance of previous CD during labor, number of previous CDs, the inter- 
Table 1. Baseline characteristics.

\begin{tabular}{cccc}
\hline & Group A ( $=$ 69) & Group B (n = 20) & p-value \\
\hline Maternal age (year) & $34.5 \pm 0.6$ & $33.4 \pm 1.1$ & 0.24 \\
Parity & $1.70 \pm 0.1$ & $0.55 \pm 0.2$ & $<0.001$ \\
Gestational age at birth (wks) & $37.9 \pm 0.1$ & $38.1 \pm 0.1$ & 0.45 \\
Neonatal weight at birth (g) & $2823 \pm 42.2$ & $2957 \pm 78.4$ & 0.10 \\
Number of previous CDs & $1(1-4)$ & & \\
Interval time from the last CD (month) & $35(14-156)$ & & \\
\hline
\end{tabular}

CD: Cesarean delivery.

Table 2. LUS thickness in each group.

\begin{tabular}{cccc}
\hline & Group A $(\mathrm{n}=69)$ & Group B (n= 20) & p-value \\
\hline mLUS $(\mathrm{mm})$ & $1.50(0.40-3.83)$ & $2.75(0.77-10.7)$ & $<0.0001$ \\
fLUS $(\mathrm{mm})$ & $4.07(1.53-7.03)$ & $5.37(3.30-17.4)$ & 0.0023 \\
\hline
\end{tabular}

mLUS: myometrial lower uterine segment; fLUS: full thickness lower uterine segment.

Table 3. Correlation between LUS thickness and LUS grade.

\begin{tabular}{|c|c|c|c|}
\hline & Grade III, IV $(\mathrm{n}=8)$ & Grade I, II (n = 81) & p-value \\
\hline mLUS (mm) & $0.67(0.40-1.47)$ & $2.07(0.40-10.7)$ & $<0.0001$ \\
\hline fLUS (mm) & $2.52(1.60-4.30)$ & $4.37(1.53-17.4)$ & 0.0005 \\
\hline
\end{tabular}

mLUS: myometrial lower uterine segment; fLUS: full thickness lower uterine segment.

Table 4. Univariate logistic regression analysis to identify the risk factors for grade III-IV.

\begin{tabular}{|c|c|c|c|}
\hline Variables & OR & $95 \% \mathrm{CI}$ & p-value \\
\hline Maternal age & 1.020 & $0.881-1.206$ & 0.803 \\
\hline Parity & 1.367 & $0.670-2.634$ & 0.370 \\
\hline The interpregnancy time from last CD & 0.992 & $0.951-1.021$ & 0.609 \\
\hline Gestational age at birth & 1.126 & $0.276-3.586$ & 0.857 \\
\hline Neonatal weight at birth & 1.000 & $0.998-1.002$ & 0.762 \\
\hline fLUS & 0.198 & $0.059-0.481$ & $<0.0001$ \\
\hline mLUS & 0.031 & $0.0016-0.219$ & $<0.0001$ \\
\hline Number of previous CDs & 1.780 & $0.819-3.903$ & 0.142 \\
\hline Previous CD during labor & 0.480 & $0.025-3.034$ & 0.476 \\
\hline
\end{tabular}

OR: odds ratio; CI: confidence interval; CD: cesarean delivery; mLUS: myometrial lower uterine segment; fLUS: full thickness lower uterine segment.

pregnancy time from the last CD, and LUS grades III/IV. Receiver operating characteristic analysis showed that the LUS thickness for predicting LUS grades III/IV was $0.97 \mathrm{~mm}$ of mLUS (area under the curve, $0.9105 ; \mathrm{p}=$ 0.0049 ) and $3.13 \mathrm{~mm}$ of fLUS (area under the curve, 0.8773; $\mathrm{p}=0.0021$ ) (Figure 2), having a sensitivity of $87.5 \%$, a specificity of $87.7 \%$, a positive predictive value of $41.2 \%$, and a negative predictive value of $98.6 \%$ in mLUS measurement and a sensitivity of $75.0 \%$, a specificity of $91.4 \%$, a positive predictive value of $46.2 \%$, and a negative predictive value of $97.4 \%$ in fLUS measurement (Table 5).

\section{Discussion}

Our study showed that ultrasonographic measurements of mLUS and fLUS at term were significantly different between the two study groups. We also demonstrated that mLUS and fLUS thickness in women with a prior CD were strong predictors for uterine rupture or uterine dehiscence according to univariate analysis, with cutoff values of 0.97 and $3.13 \mathrm{~mm}$, respectively, where mLUS and fLUS thickness were found to be almost equivalent 
mLUS and LUS grade (III-IV) prediction

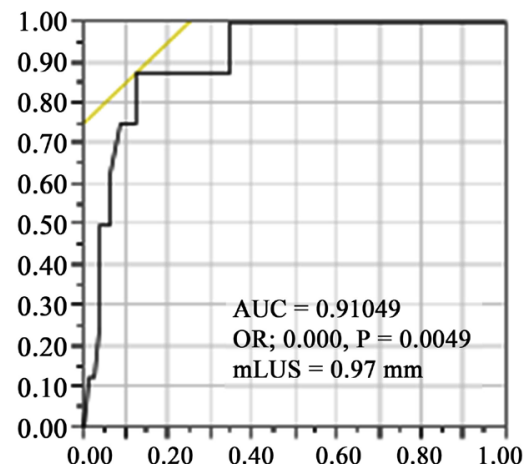

fLUS and LUS grade (III-IV) prediction

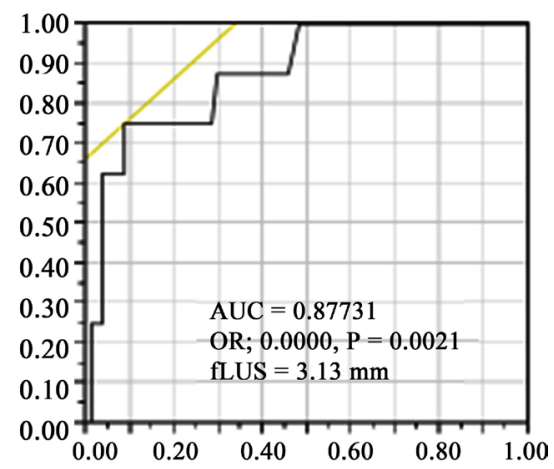

Figure 2. LUS thickness and LUS grade prediction by receiver operating characteristic analysis.

Table 5. mLUS and fLUS thickness and LUS grade.

Sensitivity $87.5 \%$, specificity $87.7 \%$, PPV $41.2 \%$, NPV 98.6\%

\begin{tabular}{ccc}
\hline \multirow{2}{*}{ mLUS } & \multicolumn{3}{c}{ LUS grade } \\
\cline { 2 - 3 } & III, IV & I, II \\
\hline $0.97 \mathrm{~mm}$ & 7 & 10 \\
$>0.97 \mathrm{~mm}$ & 1 & 71 \\
\hline
\end{tabular}

Sensitivity 75.0\%, specificity 91.4\%, PPV 46.2\%, NPV 97.4\%

\begin{tabular}{ccc} 
fLUS & \multicolumn{3}{c}{ LUS grade } \\
\cline { 2 - 3 } & III, IV & I, II \\
\hline $3.13 \mathrm{~mm}$ & 6 & 7 \\
$>3.13 \mathrm{~mm}$ & 2 & 74 \\
\hline
\end{tabular}

mLUS: myometrial lower uterine segment; fLUS: full thickness lower uterine segment.

assessed by AUC of receiver operating characteristic analysis. In our study, measuring the mLUS, which is more technically difficult, did not add anything to the positive predictive value. The range of error during the sonographic measurement must be minimized because many factors can affect the LUS measurement (contractile state of the uterus, displacement of amniotic fluid, fetal movement and position, operator's pressure through the transducer during the examination, and fullness of the bladder) [11]. Measuring only fLUS may be sufficient in estimating the risk of uterine dehiscence. However, the study had several limitations. First, it was a small cohort study and could have led to type 2 errors. A second limitation was that it might not be possible to analyze interactions between risk factors with only 8 cases of LUS grade III/IV observed.

Dehiscence of the LUS is a life-threatening event. Previous studies have found that patients with an LUS thickness of 2.5 or $3.5 \mathrm{~mm}$ nave the highest risk of uterine rupture [10] [12] [13]. LUS thickness greater than $4.45 \mathrm{~mm}$ was found to be protective against uterine rupture [10]. Recent research demonstrated that LUS thickness less than $2.3 \mathrm{~mm}$ is associated with a higher risk of complete uterine rupture [13]. To date, two meta-analyses of LUS measurement have been published. Jastrow et al. [17] conducted a meta-analysis of 12 articles on LUS thickness and risk of uterine scar defect and showed a strong association between the degrees of LUS thinning and the risk of uterine defects. Kok et al. [18] in their meta-analysis of 21 studies reported that a full LUS thickness cutoff of $3.1-5.1 \mathrm{~mm}$ and a myometrium thickness cutoff of $2.1-4.0 \mathrm{~mm}$ provided a strong negative predictive value for the occurrence of a defect during TOLAC. A myometrium thickness cutoff between 0.6 and $2.0 \mathrm{~mm}$ provided a strong positive predictive value for the occurrence of a defect. However, an ideal LUS thickness cutoff value that could be used in clinical practice in women with a scarred uterus could not be defined by these two meta-analyses because of the heterogeneity of the studies. Another concern was that the many small studies were inclined to overestimate the prediction [19], which is the case with our study. Furthermore, there were various definitions of uterine defects among the studies, ranging from thinning to complete rupture. Another important factor was that there was no consensus among the studies regarding which layers of the LUS 
should be measured, or by which route, transabdominal or transvaginal. Consequently, large cohort studies are absolutely necessary in which the LUS measurements are not disclosed to the attending physicians until after the delivery.

We observed six cases of LUS grade III and two cases of LUS grade IV, where seven cases had less than cutoff value of mLUS and six cases had less than cutoff value of fLUS. Contradictory to other studies, all cases of LUS grade III or IV occurred when the interpregnancy time from the most recent previous CD was more than 18 months [20]-[22]. This time was reported to be the minimum time necessary for adequate activation and for completion of a slow healing process [23]. However, there were only eight cases with an interpregnancy time of less than 18 months in our study population, and a large number of retrospective studies showed that a short time interval was not a risk factor for major maternal and neonatal complications such as uterine rupture [24].

\section{Conclusion}

An ideal screening test to predict uterine dehiscence would require high levels of both sensitivity and specificity $(\geq 90 \%)$. If such a test were to become available, it is very likely that this would influence medical decisionmaking, through the accurate selection of women with a previous CD unlikely to have uterine rupture and therefore suitable for a TOLAC, as opposed to women with a previous CD likely to have a uterine rupture and therefore suitable for repeat CD. Sonographic measurements of LUS at term may be a feasible and reliable method to predict uterine rupture or uterine dehiscence in women with a prior CD.

\section{References}

[1] Niino, Y. (2011) The Increasing Cesarean Rate Globally and What We Can Do about It. Bioscience Trends, 5, $139-150$. http://dx.doi.org/10.5582/bst.2011.v5.4.139

[2] Martin, J.A., Hamilton, B.E., Ventura, S.J., Osterman, M.J. and Mathews, T.J. (2013) Births: Final Data for 2011. National Vital Statistics Reports, 62, 1-69, 72.

[3] Getahun, D., Oyelese, Y., Salihu, H.M. and Ananth, C.V. (2006) Previous Cesarean Delivery and Risks of Placenta Previa and Placental Abruption. Obstetrics and Gynecology, 107, 771-778. http://dx.doi.org/10.1097/01.AOG.0000206182.63788.80

[4] Bowman, Z.S., Eller, A.G., Bardsley, T.R., Greene, T., Varner, M.W. and Silver, R.M. (2014) Risk Factors for Placenta Accreta: A Large Prospective Cohort. American Journal of Pathology, 31, 799-804.

[5] National Institutes of Health Consensus Development Conference Statement (2010) Vaginal Birth after Cesarean: New Insights March 8-10, 2010. Obstetrics and Gynecology, 115, 1279-1295.

[6] American College of Obstetricians and Gynecologists (2010) ACOG Practice Bulletin No. 115: Vaginal Birth after Previous Cesarean Delivery. Obstetrics and Gynecology, 116, 450-463. http://dx.doi.org/10.1097/AOG.0b013e3181eeb251

[7] McMahon, M.J., Luther, E.R., Bowes Jr, W.A. and Olshan, A.F. (1996) Comparison of a Trial of Labor with an Elective Second Cesarean Section. New England Journal of Medicine, 335, 689-695. http://dx.doi.org/10.1056/NEJM199609053351001

[8] Lydon-Rochelle, M., Holt, V.L., Easterling, T.R. and Martin, D.P. (2001) Risk of Uterine Rupture during Labor among Women with a Prior Cesarean Delivery. New England Journal of Medicine, 345, 3-8. http://dx.doi.org/10.1056/NEJM200107053450101

[9] Rozenberg, P., Goffinet, F., Phillippe, H.J. and Nisand, I. (1996) Ultrasonographic Measurement of Lower Uterine Segment to Assess Risk of Defects of Scarred Uterus. Lancet, 347, 281-284. http://dx.doi.org/10.1016/S0140-6736(96)90464-X

[10] Rozenberg, P., Goffinet, F., Philippe, H.J. and Nisand, I. (1997) Echographic Measurement of the Inferior Uterine Segment for Assessing the Risk of Uterine Rupture. Journal de Gynécologie Obstétrique et Biologie de la Reproduction, 26, 513-519.

[11] Asakura, H., Nakai, A., Ishikawa, G., Suzuki, S. and Araki, T. (2000) Prediction of Uterine Dehiscence by Measuring Lower Uterine Segment Thickness Prior to the Onset of Labor: Evaluation by Transvaginal Ultrasonography. Journal of Nippon Medical School, 67, 352-356. http://dx.doi.org/10.1272/jnms.67.352

[12] Gotoh, H., Masuzaki, H., Yoshida, A., Yoshimura, S., Miyamura, T. and Ishimaru, T. (2000) Predicting Incomplete Uterine Rupture with Vaginal Sonography during the Late Second Trimester in Women with Prior Cesarean. Obstetrics \& Gynecology, 95, 596-600. http://dx.doi.org/10.1016/S0029-7844(99)00620-1

[13] Bujold, E., Jastrow, N., Simoneau, J., Brunet, S. and Gauthier, R.J. (2009) Prediction of Complete Uterine Rupture by 
Sonographic Evaluation of the Lower Uterine Segment. American Journal of Obstetrics \& Gynecology, 201, 320.e1320.e6. http://dx.doi.org/10.1016/j.ajog.2009.06.014

[14] Ginsberg, Y., Goldstein, I., Lowenstein, L. and Weiner, Z. (2013) Measurements of the Lower Uterine Segment during Gestation. Journal of Clinical Ultrasound, 41, 214-217. http://dx.doi.org/10.1002/jcu.22023

[15] Cheung, V.Y., Constantinescu, O.C. and Ahluwalia, B.S. (2004) Sonographic Evaluation of the Lower Uterine Segment in Patients with Previous Cesarean Delivery. Journal of Ultrasound in Medicine, 23, 1441-1447.

[16] Qureshi, B., Inafuku, K., Oshima, K., Masamoto, H. and Kanazawa, K. (1997) Ultrasonographic Evaluation of Lower Uterine Segment to Predict the Integrity and Quality of Cesarean Scar during Pregnancy: A Prospective Study. Tohoku Journal of Experimental Medicine, 183, 55-65. http://dx.doi.org/10.1620/tjem.183.55

[17] Jastrow, N., Chaillet, N., Roberge, S., Morency, A.M., Lacasse, Y. and Bujold, E. (2010) Sonographic Lower Uterine Segment Thickness and Risk of Uterine Scar Defect: A Systematic Review. Journal of Obstetrics and Gynaecology Canada, 32, 321-327.

[18] Kok, N., Wiersma, I.C., Opmeer, B.C., de Graaf, I.M., Mol, B.W. and Pajkrt, E. (2013) Sonographic Measurement of Lower Uterine Segment Thickness to Predict Uterine Rupture during a Trial of Labor in Women with Previous Cesarean Section: A Meta-Analysis. Ultrasound in Obstetrics \& Gynecology, 42, 132-139. http://dx.doi.org/10.1002/uog.12479

[19] Leeflang, M.M., Moons, K.G., Reitsma, J.B. and Zwinderman, A.H. (2008) Bias in Sensitivity and Specificity Caused by Data-Driven Selection of Optimal Cut-Off Values: Mechanisms, Magnitude, and Solutions. Clinical Chemistry, 54, 729-737. http://dx.doi.org/10.1373/clinchem.2007.096032

[20] Shipp, T.D., Zelop, C.M., Repke, J.T., Cohen, A. and Lieberman, E. (2001) Interdelivery Interval and Risk of Symptomatic Uterine Rupture. Obstetrics \& Gynecology, 97, 175-177. http://dx.doi.org/10.1016/S0029-7844(00)01129-7

[21] Bujold, E. and Gauthier, R.J. (2010) Risk of Uterine Rupture Associated with an Interdelivery Interval between 18 and 24 Months. Obstetrics \& Gynecology, 115, 1003-1006. http://dx.doi.org/10.1097/AOG.0b013e3181d992fb

[22] Gizzo, S., Zambon, A., Saccardi, C., Patrelli, T.S., Di Gangi, S., Carrozzini, M., Bertocco, A., Capobianco, G., D’Antona, D. and Nardelli, G.B. (2013) Effective Anatomical and Functional Status of the Lower Uterine Segment at Term: Estimating the Risk of Uterine Dehiscence by Ultrasound. Fertility and Sterility, 99, 496-501. http://dx.doi.org/10.1016/j.fertnstert.2012.10.019

[23] Landon, M.B. (2010) Predicting Uterine Rupture in Women Undergoing Trial of Labor after Prior Cesarean Delivery. Seminars in Perinatology, 34, 267-271. http://dx.doi.org/10.1053/j.semperi.2010.03.005

[24] Kessous, R. and Sheiner, E. (2013) Is There an Association between Short Interval from Previous Cesarean Section and Adverse Obstetric and Perinatal Outcome? The Journal of Maternal-Fetal \& Neonatal Medicine, 26, 1003-1006. http://dx.doi.org/10.3109/14767058.2013.765854 